\title{
Uterine Myometrial Preischemia: Yet Another Physiological Change of Pregnancy!
}

\author{
Kusum Jashnani ${ }^{1} \quad$ Meherrituja Palve ${ }^{2}$ \\ ${ }^{1}$ Department of Pathology, TNMC \& BYL Nair Ch. Hospital, Mumbai, \\ Maharashtra, India \\ 2 Department of Pathology, KJSMC and Research Centre, Mumbai, \\ Maharashtra, India \\ J Lab Physicians 2022;14:157-163.
}

Address for correspondence Meherrituja Palve, MBBS, MD, Department of Pathology, KJSMC and Research Centre, Mumbai 400022, Maharashtra, India (e-mail: meherutujapalve@yahoo.co.in).

\begin{abstract}
Keywords

- pregnancy

- myometrial hypertrophy

- preischemia

- vascular remodeling

- cervical wall hemorrhages

Background The uterus shows tremendous increase in size during pregnancy to nurture the fetus within it. It may show a spectrum of physiological changes or pathological lesions that may affect the pregnancy favorably or adversely. The main purpose of our study was to analyze the physiological changes in the uterus during pregnancy and the postpartum period, thereby gaining deeper knowledge.

Materials and Methods We studied a total of 152 uterine specimens obtained from obstetric hysterectomies and maternal autopsies for the presence or absence of normal physiological changes. As a control group, an equal number of surgical uterine specimens received for abnormal uterine bleeding were studied.

Results Decidual change was observed from 6 weeks of gestation to 16 days postpartum. It was partially deficient to absent in four cases of placenta accreta. Myometrial hypertrophy was seen in 150 cases $(98.68 \%$ ) from 8 weeks of gestation till 30 days postpartum period. Vascular remodeling was partially deficient to absent in eight cases of pregnancy-induced hypertension. Preischemia of myometrial fibers was an unexpected finding noticed from 20 weeks of gestation to 16 days postpartum in 131 cases $(86.18 \%)$. Cervical wall hemorrhages were seen in $84.84 \%$ cases of vaginal delivery and in only $17.64 \%$ cases of lower segment cesarean section.

Conclusion Ours is the first study to describe the duration of the routine physiological uterine changes during pregnancy. The relationship between cervical wall hemorrhages and vaginal delivery as well as between myometrial preischemia and gestational age, both being normal physiologic findings, was found to be statistically significant.
\end{abstract}

\section{Key Messages}

Understanding physiological changes has always given answers to the complex questions of the pathology of various conditions.
In this study, we have analyzed the uteri for various physiological changes of pregnancy and puerperium. We found some novel physiological changes like preischemia and positive correlation between vaginal delivery and cervical wall hemorrhages. published online August 24, 2021
DOI https://doi.org/ 10.1055/s-0041-1734016. ISSN 0974-2727. (c) 2021. The Indian Association of Laboratory Physicians. All rights reserved.

This is an open access article published by Thieme under the terms of the Creative Commons Attribution-NonDerivative-NonCommercial-License, permitting copying and reproduction so long as the original work is given appropriate credit. Contents may not be used for commercial purposes, or adapted, remixed, transformed or built upon. (https://creativecommons.org/ licenses/by-nc-nd/4.0/)

Thieme Medical and Scientific Publishers Pvt. Ltd., A-12, 2nd Floor, Sector 2, Noida-201301 UP, India 
A pathologist should be aware of these physiological changes lest it may be mistaken to be pathological. Also, further ultrastructural studies may help in unveiling the exact mechanism of the adaptive powers of the uteri that may possibly have therapeutic applications someday.

\section{Introduction}

The female genital tract, especially uterus, undergoes extensive physiological and structural adaptations to pregnancy because it has to contain and nurture the growing fetus. It increases from the size of a small pear in its nonpregnant state to accommodate a full-term baby at 40 weeks of gestation (-Fig. 1A, B). The increase in uterine size is achieved by uterine smooth muscle hypertrophy and hyperplasia. Deep placentation in the uterus requires physiologic transformation of the spiral arteries into uteroplacental vessels. Absent or inadequate physiological changes of pregnancy underlie the defective fetomaternal interactions resulting in preeclampsia, fetal intrauterine growth retardation, antepartum, and postpartum hemorrhage.

In the majority of abnormal pregnancies, for obvious reasons, the uterus is not available for histopathological

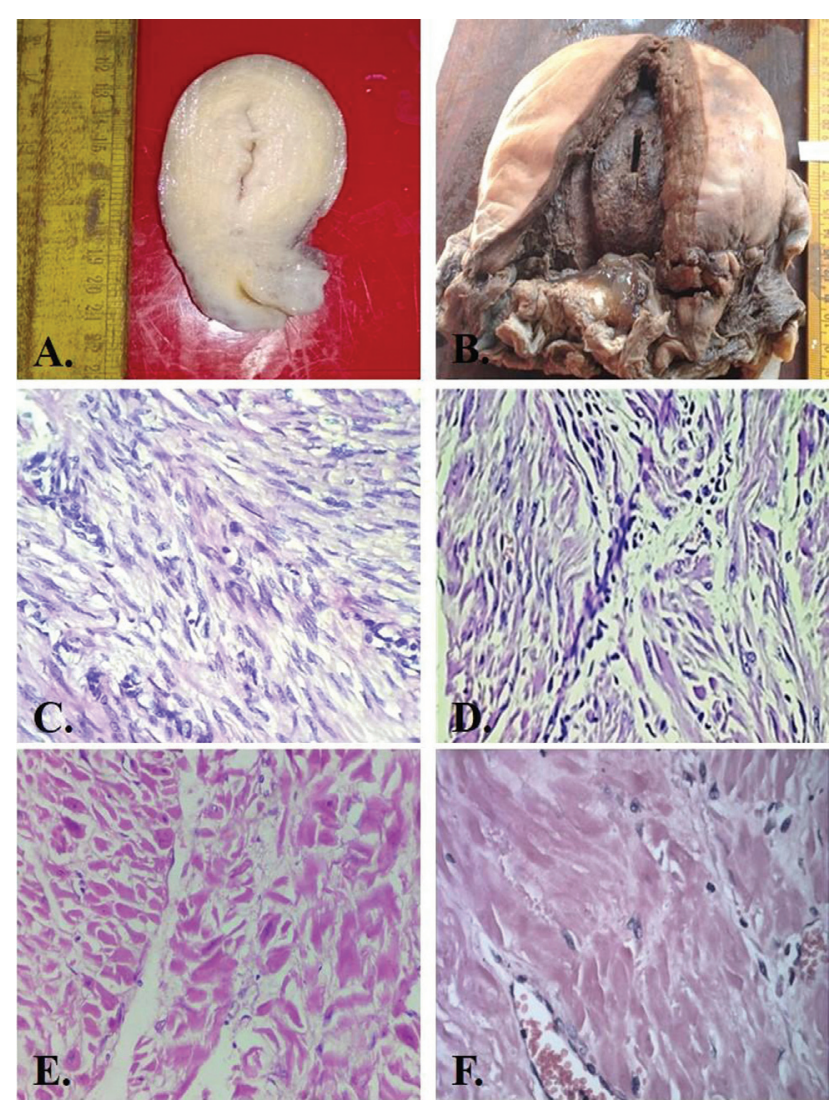

Fig. 1 Normal uterus with slit like endometrial cavity. (A) Full-term postpartum uterus with marked increase in size showing dilated endometrial cavity. (B) Myometrium in nonpregnant uterus showing normal sized smooth muscle fibers with basophilic elongated nuclei. (C) $400 \times$, H\&E; Mild increase in smooth muscle fiber size and preserved basophilic elongated nuclei in a case of 15 -week gestational age-hypertrophy only. (D) 400×, H\&E; Increase in size of smooth muscle fibers with inconspicuous to absent nuclei in $28(\mathrm{E})$ and 32 weeks (F) of gestational age-hypertrophy with preischemia; $400 \times, \mathrm{H} \& \mathrm{E}$. study so that deductions about the disorder in question must be made from the delivered placenta and, in cases of perinatal death, from the autopsy studies of the neonate. The uterus involutes postpartum and any pregnancy-associated defect on the maternal side of the placenta disappears in the process and may not recur in subsequent pregnancies. The technique of placental bed biopsy and autopsies in cases of maternal death can provide some knowledge; however, the emphasis is more upon the cause of death and less upon the elucidation of the pathology of the condition leading to the fatality. ${ }^{1-4}$

Uterine specimens obtained in the cases of obstetric hysterectomies (OHs) are also a useful source for studying the adaptations of pregnant state and their disorders. However, majority of the studies on the OHs focus more on the indications, management, and complications of the procedure than on the pathophysiological changes in the uterus.

The uterus may show a spectrum of physiological changes or pathological lesions that may affect the pregnancy favorably or adversely. These lesions may be symptomatic or just incidental findings. The main purpose of our study is to analyze the physiological changes in the uterus during pregnancy and the postpartum period, thereby gaining deeper knowledge.

\section{Materials and Methods}

This was a retrospective and prospective observational 3year study. Institutional Ethics Committee approval was obtained. One-hundred fifty-two consecutive uteri received in the form of $\mathrm{OHs}$ and uteri examined/removed during autopsies performed on maternal deaths were included in the study. Medicolegal autopsies on maternal death and uteri removed after 42 days of delivery were excluded from the study. Adnexa and placental findings were not included.

One-hundred fifty-two uteri of nonpregnant females received in surgical pathology laboratory in the form of hysterectomies performed due to abnormal uterine bleeding were taken as control group. Cases of uterine or cervical cancers were excluded in this group. Relevant clinical data, gross examination findings of the uterine specimens, etc. were taken from the records. Hematoxylin and eosin-stained slides of the specimens were reviewed for the microscopic findings. Physiological changes sought for in the specimens on histopathological examination were as follows:

- Decidual changes in the endometrium.

- Hypertrophy of the smooth muscle fibers.

- Preischemic changes of the muscle fibers-this was a novel finding noticed.

- Trophoblastic invasion of decidua and myometrium.

- Transformation of the spiral arteries into uteroplacental vessels by remodeling.

- Areas of hemorrhage and congested blood vessels in the cervical wall.

- Absence or inadequacy of the above physiological changes was looked for. 


\section{Statistical Test}

Qualitative data was represented in the form of frequency and percentage.

Association between qualitative variables was assessed by chi-squared test for all $2 * 2$ tables and Fisher's exact test for all $2^{*} 2$ tables where $p$-value of chi-squared test is not valid due to small counts.

\section{Results}

Minimum age at presentation in the study group was 18 years and maximum age at presentation was 40 years. About $71 \%$ of cases ( $n=108$ ) belonged to 21 to 30 years age group. The 152 uterine specimens were obtained from OHs $(n=37)$ and from maternal postmortems $(n=115)$. Most patients in the control group were in the age group of 31 to 40 years with minimum age at presentation being 29 years and maximum age at presentation being 45 years.

About $22 \%$ of the gravid patients presented in the third trimester and $58 \%$ patients $(n=88)$ presented in the postpartum period. In the autopsy group ( - Table 1 ), maximum cases $(n=45 ; 60.81 \%)$ were delivered vaginally. In the $\mathrm{OH}$ group (-Table 2 ), most of the females were delivered by lower segment cesarean section (LSCS; $n=17 ; 45.94 \%$ ) with the indications of $\mathrm{OH}$ as mentioned in - Table 3. Causes of maternal deaths ( - Table 4 ) and physiological changes in the uterus ( - Table 5 ) were sought for.

Decidual change in the endometrium was seen from 6 weeks of pregnancy to 16 days postpartum. Decidual change was partial to absent in four cases of placenta accreta $(n=11)$. Five cases in the late postpartum period also did not show the decidual change. None of the cases in the control group showed decidual change.

Myometrial hypertrophy (increase in the myometrial smooth muscle size) was seen from 8 weeks of pregnancy to 30 days postpartum ( $\mathbf{- F i g}$. 1D, E, F). Cases in the control group showed normal sized smooth muscle fibers (-Fig. 1C).

Preischemia, seen in the form of very lightly stained to inconspicuous nuclei in the hypertrophied myometrial smooth muscle fibers, has not been documented in the literature. In our study, preischemia was seen from 20 weeks of pregnancy and was seen till 16-day postpartum (- Fig. 1E, F). It was absent in 21 cases that included all the six cases of

Table 1 Mode of delivery in postpartum cases $(n=74)$ in autopsy group

\begin{tabular}{|l|l|l|}
\hline & No. of cases & $\%$ \\
\hline Delivered vaginally & 45 & 60.81 \\
\hline Delivered by LSCS & 17 & 22.98 \\
\hline Aborted & 8 & 10.81 \\
\hline Expelled spontaneously after death & 2 & 2.70 \\
\hline Prior obstetric hysterectomy & 2 & 2.70 \\
\hline Total & 74 & 100 \\
\hline
\end{tabular}

Abbreviation: LSCS, lower segment cesarean section. first trimester. None of the cases of the control group showed evidence of preischemic changes.

Remodeling of vessels can be studied when sections are taken from the placental bed. In our study, remodeling of vessels was seen in $56.58 \%$ cases $(n=86)$ from 12 weeks onwards. At 12 weeks, the remodeling was seen in the decidual vessels alone. Remodeling of the superficial myometrial vessels was seen in the second trimester at around 18 to 20 weeks (-Fig. 2A). Vascular remodeling was deficient to absent in eight cases of pregnancy-induced hypertension (PIH; - Fig. 2C, D; - Table 6). Remodeling could not be appreciated in $36.18 \%$ cases $(n=55)$ due to sections not being taken from representative placental bed.

Intermediate trophoblasts (implantation type) were seen invading the decidua and the superficial myometrium in $63.82 \%$ cases $(n=97)$. They could not be seen in $36.18 \%$ cases $(n=55)$ as the sections were not taken from the placental bed.

The mode of delivery ( - Table 7 ) had a significant impact on the cervical wall. Extensive cervical wall hemorrhages were seen in $84.84 \%$ cases $(n=56)$ of vaginal deliveries (-Fig. 2F) and only in $17.64 \%$ cases $(n=6)$ of LSCS ( $p$ value $<0.0000001$ ).

Table 2 Mode of delivery in obstetric hysterectomies $(n=37)$

\begin{tabular}{|l|l|l|}
\hline Mode of delivery & No. of cases & $\%$ \\
\hline Vaginal & 4 & 10.82 \\
\hline $\begin{array}{l}\text { Assisted vaginal deliveries } \\
\text { (vacuum and forceps) }\end{array}$ & 3 & 8.11 \\
\hline Lower segment caesarean section & 17 & 45.95 \\
\hline $\begin{array}{l}\text { Simultaneous exploration and } \\
\text { obstetric hysterectomy }\end{array}$ & 9 & 24.32 \\
\hline Aborted & 2 & 5.40 \\
\hline Medical termination of pregnancy & 2 & 5.40 \\
\hline Total & 37 & 100 \\
\hline
\end{tabular}

Table 3 Indications of obstetric hysterectomies

\begin{tabular}{|l|l|l|}
\hline Indication & No. of cases & $\%$ \\
\hline PPH & 19 & 51.35 \\
\hline Rupture & 9 & 24.32 \\
\hline Placenta accreta & 3 & 8.10 \\
\hline Abruptio placentae & 2 & 5.40 \\
\hline Suture site hematoma & 1 & 2.70 \\
\hline Ectopic in cervix & 1 & 2.70 \\
\hline Hydatidiform mole & 1 & 2.70 \\
\hline Pelvic collection post LSCS & 1 & 2.70 \\
\hline Total & 37 & 100 \\
\hline
\end{tabular}

Abbreviations: LSCS, lower segment cesarean section; PPH, postpartum hemorrhage. 
Table 4 Final causes of death in autopsy cases

\begin{tabular}{|c|c|c|c|}
\hline & & No. of cases & $\%$ \\
\hline & PIH related & 12 & 10.42 \\
\hline Direct causes of maternal deaths & Puerperal sepsis & 3 & 2.60 \\
\hline \multirow[t]{6}{*}{$(n=25,21.74 \%)$} & DIC & 3 & 2.60 \\
\hline & $\mathrm{PPH}$ & 2 & 1.73 \\
\hline & Cortical venous sinus thrombosis & 2 & 1.73 \\
\hline & Acute fatty liver of pregnancy & 1 & 0.86 \\
\hline & Abruptio placentae & 1 & 0.86 \\
\hline & Rupture & 1 & 0.86 \\
\hline Indirect & 1. Infection & 38 & 33.04 \\
\hline \multirow{27}{*}{$\begin{array}{l}\text { Causes of maternal deaths } \\
(n=90,78.26 \%)\end{array}$} & I) Hepatitis E & 20 & 17.39 \\
\hline & II) Hepatitis B & 1 & 0.86 \\
\hline & III) $\mathrm{TB}$ & 10 & 8.69 \\
\hline & IV) Dengue & 3 & 2.60 \\
\hline & V) H1N1 & 2 & 1.73 \\
\hline & VI) Malaria & 1 & 0.86 \\
\hline & VII) Neurocysticercosis & 1 & 0.86 \\
\hline & 2. Pulmonary & 16 & 13.91 \\
\hline & I) Bronchopneumonia & 7 & 5.90 \\
\hline & II) ARDS & 5 & 4.33 \\
\hline & III) Pulmonary edema & 3 & 2.59 \\
\hline & IV) Aspiration pneumonitis & 1 & 0.86 \\
\hline & 3. Cardiovascular & 15 & 13.04 \\
\hline & I) Rheumatic heart disease & 9 & 7.82 \\
\hline & II) Dilated cardiomyopathy & 5 & 4.34 \\
\hline & III) Acute coronary insufficiency with IHD & 1 & 0.86 \\
\hline & 4. Liver & 8 & 6.95 \\
\hline & I) Hepatic necrosis-cause unknown & 6 & 5.21 \\
\hline & II) Cirrhosis & 1 & 0.86 \\
\hline & III) Acute hepatitis & 1 & 0.86 \\
\hline & 5. Septicemia & 6 & 5.21 \\
\hline & 6. Bilateral renal cortical necrosis & 2 & 1.73 \\
\hline & 7. Hepatorenal failure & 1 & 0.86 \\
\hline & 8. Metabolic & 1 & 0.86 \\
\hline & 9. Subarachnoid hemorrhage & 1 & 0.86 \\
\hline & 10. Anemia & 1 & 0.86 \\
\hline & 11. Gl bleeding & 1 & 0.86 \\
\hline
\end{tabular}

Abbreviations: ARDS, acute respiratory distress syndrome; DIC, disseminated intravascular coagulation; GI, gastrointestinal; IHD, ischemic heart disease; PIH, pregnancy-induced hypertension; PPH, postpartum hemorrhage; TB, tuberculosis.

\section{Discussion}

In this study, we have examined the uteri in detail for physiologic changes during pregnancy and postpartum period. The uterine specimens included those removed during maternal death autopsies and those received as OHs. Presence or absence of normal physiologic uterine wall changes of decidualization, uterine myometrial hypertrophy, and preischemia as well as vascular remodeling was studied in each and every case. 
Table 5 Physiological changes in the study group

\begin{tabular}{|c|c|c|c|c|c|c|c|c|}
\hline & $\begin{array}{l}\text { Seen in } \\
\text { (total-152 } \\
\text { cases) }\end{array}$ & $\%$ & Not seen in & $\%$ & $\begin{array}{l}\text { Partial change } \\
\text { or deficient }\end{array}$ & $\%$ & $\begin{array}{l}\text { Section not } \\
\text { representative }\end{array}$ & $\%$ \\
\hline $\begin{array}{l}\text { Decidual change in } \\
\text { endometrium }\end{array}$ & 143 & 94.08 & 6 & 3.95 & 3 & 1.97 & - & - \\
\hline Myometrial hypertrophy & 150 & 98.68 & 2 & 1.32 & - & - & - & - \\
\hline Preischemia & 131 & 86.18 & 21 & 13.82 & - & - & - & - \\
\hline Remodeling of vessels & 86 & 56.58 & 8 & 5.27 & 3 & 1.97 & 55 & 36.18 \\
\hline Intermediate trophoblasts & 97 & 63.82 & - & - & - & - & 55 & 36.18 \\
\hline
\end{tabular}

Table 6 Vascular remodeling in PIH

\begin{tabular}{|l|l|l|l|}
\hline PIH & & No. of cases & $\%$ \\
\hline Remodeling present & & 5 & 31.25 \\
\hline Remodeling absent & & 6 & 37.5 \\
\hline Partial remodeling & 2 & 12.5 \\
\hline Sections not representative & & 3 & 18.75 \\
\hline
\end{tabular}

Abbreviation: PIH, pregnancy-induced hypertension.

The first signs of decidualization can be seen as early as 23rd day of the menstrual cycle (ten days after the peak of the luteinizing hormone surge), when the spiral arterioles of the endometrium first become prominent. Complete decidualization is, however, not evident before pregnancy is well established. ${ }^{1}$ In early pregnancy, the decidua begins to thicken, eventually attaining a depth of 5 to $10 \mathrm{~mm}$. Later in pregnancy, the decidua becomes thinner, presumably because of pressure exerted by the expanding uterine contents. ${ }^{5}$ of the total 152 cases, decidualization of the endometrium was seen in 143 cases (94.07\%) from 6 weeks of gestation to 16 days postpartum. Partial to absent decidualization was a finding in four cases (2.63\%) of placenta accreta. Decidualization was not seen after 16 days of postpartum period in five cases.

Hypertrophy refers to an increase in the size of cells that result in an increase in the size of the affected organ. It is a reversible physiological or pathological adaptation to changes in the environment. In the uterus, massive physiological growth in pregnancy is an example of hormoneinduced enlargement that results mainly from hypertrophy of muscle fibers. Thus, myometrial hypertrophy was seen in 150 cases (98.68\%) from 8 weeks of gestation till 30 days postpartum period. It was not seen after 30 days postpartum period suggesting that it being a reversible change, the muscle fibers would have returned to their normal state in the absence of the stimulus that is provided otherwise by the growing fetus and the increasing hormone levels. Increase in uterine size in pregnancy is also supposed to be due to myometrial hyperplasia, a finding that we were not able to substantiate in this study.

An additional finding observed in our study was of preischemia. Very lightly stained to inconspicuous nuclei in the hypertrophied muscle fibers (similar to karyolysis that is normally seen in coagulative necrosis due to ischemia) were referred to as preischemic changes. This change was seen involving almost 80 to $85 \%$ of the myometrial muscle fibers. There was no evidence of inflammatory reaction around these myometrial fibers. Coagulative necrosis is an irreversible change, and what we observed in our study is a reversible change as the nuclei come back to the normal after few weeks in the postpartum period.

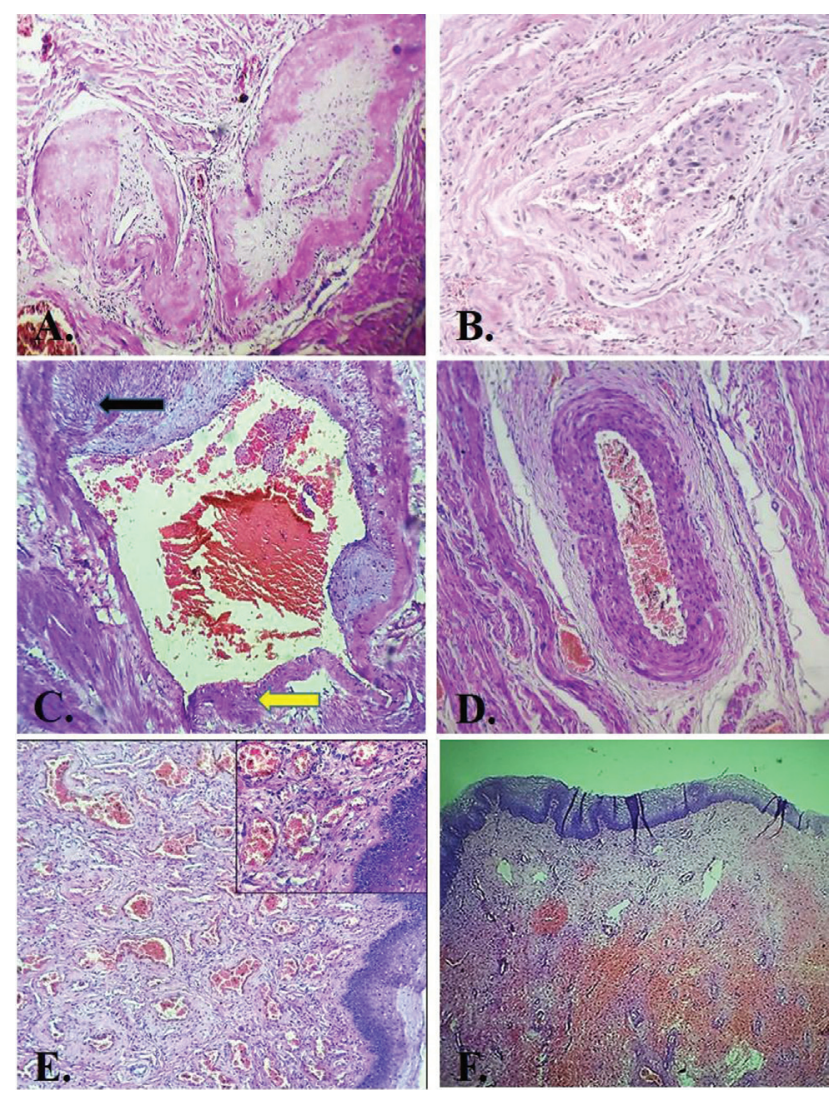

Fig. 2 Replacement of vascular muscle wall by fibrinoid materialvascular remodeling. (A) $100 \times, \mathrm{H} \& \mathrm{E}$; Intermediate trophoblasts in vessel lumen forming endovascular tufts. (B) 100×, H\&E; Partial vascular remodeling-fibrinoid deposition in part of vessel (yellow arrow) and muscle wall preserved in part (black arrow). (C) $100 \times$, $\mathrm{H} \& \mathrm{E}$; Absence of vascular remodeling in a case of pregnancy-induced hypertension. (D) $100 \times, \mathrm{H} \& \mathrm{E}$; Dilated and congested vessels in the wall of cervix in a case of lower segment cesarean section with Inset. (E) $100 \times, \mathrm{H} \& \mathrm{E}$; Extensive areas of hemorrhage in the cervical wall in a case of vaginal delivery $100 \times, H \& E(F)$. 
Table 7 Mode of delivery versus cervical wall status

\begin{tabular}{|l|l|l|l|l|l|}
\hline Mode of delivery & Total cases & Hemorrhage in the cervix & $\%$ & Congested blood vessels & $\%$ \\
\hline Vaginal & 66 & 56 & 84.84 & 50 & 75.75 \\
\hline LSCS & 34 & 6 & 17.64 & 16 & 47.05 \\
\hline Not delivered & 41 & 3 & 7.31 & 25 & 60.97 \\
\hline $\begin{array}{l}\text { Simultaneous LSCS and } \\
\text { hysterectomy }\end{array}$ & 11 & 3 & 27.27 & 2 & 18.18 \\
\hline
\end{tabular}

Abbreviation: LSCS, lower segment cesarean section.

Hence, we termed these changes as "Preischemia," as we believe it may occur due to the ischemia caused by the pressure effects of the growing fetus that compresses the uterine spiral, radial, and arcuate arteries and reduces myometrial blood flow, thus inducing starvation conditions for uterine myocytes. Whether the preischemic changes found in our study are due to an underlying autophagic process can only be confirmed by further immunohistochemical and electron microscopic studies.

In our study, preischemia was seen in 131 cases $(86.18 \%)$ from 20 weeks of gestation to 16 days postpartum. Relationship between preischemia and gestational age was found to be statistically significant, $p<0.0000001$. To the best of our knowledge, it has not been documented before in the literature. In pregnancy, uterus is responding to the mechanical strain caused by the enlarging fetus, placenta, and amniotic fluid. The effect of all this is not only increase in size of the uterus as evident by myometrial hypertrophy but also these so called preischemic changes in the myometrial smooth muscle fibers. All these efforts are taken by the human body to allow for appropriate growth of the fetus with placenta within the uterus during pregnancy. It is important to identify the nuclear changes of preischemia as a naïve pathologist may mistake it with coagulative necrosis. Flake et $\mathrm{al}^{6}$ have used a term inanosis to denote a condition of cellular inanition in uterine leiomyomas resulting from gradual nutritional deprivation. This is a process of severe atrophy (as against hypertrophy in our study), eventuating in cell death by inanosis, where blood flow is diminished by progressive stenosis of vascular lumens, resulting from the intratumoral vascular smooth muscle proliferation and fibrosis. In these cells, there is loss of nuclear staining with barely visible nuclei, and these are assumed to be nonviable. These cells are regarded as myocyte tombstones, and they are the histologic hallmark and end stage of the atrophic process is designated as inanosis.

The placental bed contains the uterine spiral arteries that supply oxygenated blood to the growing placenta and fetus. Extravillous trophoblast (EVT) cells proliferate from anchoring chorionic villi and invade the decidualized endometrium by two pathways: interstitial EVT cells invade the decidualized endometrium and inner myometrium and endovascular EVT cells invade the lumen of the spiral arteries. Some EVT become incorporated into the spiral artery wall as intramural trophoblast. The endothelium, vascular smooth muscle, and elastic lamina are destroyed and replaced by fibrinoid. By term, maternal vascular repair occurs with reendothelialization. ${ }^{7}$ EVT cell invasion is thought to be instrumental in the transformation of the muscular spiral arteries into distended, thin-walled flaccid vessels. Remodeling is necessary for a successful pregnancy. ${ }^{8}$

In our study, remodeling was seen in $56.68 \%$ cases $(n=86)$ and absent in $5.26 \%$ cases $(n=8)$ of the total sample size of 152 cases. Partial remodeling was seen in $1.97 \%$ cases $(n=3)$. In few cases, intermediate trophoblasts were present as vascular intraluminal tufts (-Fig. 2B). Placental bed was not included in $36.18 \%$ cases $(n=55)$ as it is difficult to identify in postpartum uteri, as already mentioned in previous studies also. ${ }^{9,10}$

Preeclampsia, defined by hypertension and proteinuria, which affects 3 to $7 \%$ of pregnancies, is a major contributor to maternal and fetal morbidity and mortality. ${ }^{11}$ The origin of preeclampsia is not understood but is strongly associated with failure or partial failure of spiral artery remodelling. ${ }^{7}$ Unmodified/partially modified vessels can supply high or intermittent pulses of pressure flow and so damage to the placenta results from hydrostatic stress or from changes in oxygen delivery. ${ }^{12,13}$

In our study, we had 16 cases of PIH of which vascular remodeling was present in five cases (-Table 4). These changes were absent to deficient in eight cases, which is an expected finding in PIH. ${ }^{8,14-16}$ However, in remaining three cases we could not comment on these changes as the sections were not taken from the representative placental bed. It is difficult to identify the placental bed in postpartum uteri. The relationship between partial/absent remodeling and PIH was statistically significant $(p<0.0000001)$.

The possible reason for sections from five cases showing normal physiological changes in the vessels may be due to the fact that lack of physiological change in uterine spiral arteries is not an all-or-none process, with some abnormal cases showing apparently normal remodeling. This may relate to the fact that not all cases of preeclampsia and fetal growth retardation are attributable to failed spiral artery remodelling. ${ }^{13}$

The mode of delivery had a significant effect on the cervical wall changes. Of the 66 cases that were delivered vaginally, $75.75 \%$ cases $(n=50)$ showed congested vessels in the wall of cervix and $84.84 \%$ cases $(n=56)$ showed hemorrhages in the wall. Of the total 34 cases of LSCS, cervical 
congested vessels were seen in $47.05 \%$ cases ( $n=16$; - Fig. 2E) and cervical wall hemorrhages in only $17.64 \%$ cases $(n=6)$. Thus, cervical wall hemorrhages were maximally seen in vaginally delivered cases. The gross specimen showed markedly congested to hemorrhagic cervical wall. The relationship between vaginal delivery and cervical wall hemorrhages was found to be statistically significant $(p<0.0000001)$. These hemorrhages seen in vaginal deliveries may be due to the tearing of the cervical wall vessels during the process of expulsion of the fetus. A naïve pathologist may consider the hemorrhages to be a sign of disseminated intravascular coagulation (DIC). However, we believe that the finding of hemorrhages in the cervical wall in case of vaginal deliveries should be considered a normal finding and not a sign of DIC. We would like to end by saying that this study is a morphological analysis. Further molecular and ultrastructural studies are required that may help in better understanding the mechanisms of uterine changes in pregnancy and postpartum.

The present study has been undertaken to gain in-depth knowledge of uterine physiology in pregnancy and immediate postpartum period. Literature does not provide any information on the exact duration of the normal physiological changes in pregnancy. Preischemic changes in the uterine myometrium as well as cervical wall hemorrhages are not documented in literature; therefore, these study findings are novel findings with significant implications if not already known to a naïve pathologist.

\section{Authors' Contribution}

$\mathrm{KJ}$ substantially contributed to the conception and design of the work, definition of intellectual content, and analysis and interpretation of data for the work. KJ also drafted the work and revised it critically for important intellectual content. KJ was involved in final approval of the version to be published. KJ agreed to be accountable for all aspects of the work in ensuring that questions related to the accuracy or integrity of any part of the work are appropriately investigated and resolved.

MP substantially contributed to the literature search and the acquisition, analysis, or interpretation of data for the work. MP also drafted the work or revised it critically for important intellectual content. MP was involved in final approval of the version to be published. MP agreed to be accountable for all aspects of the work in ensuring that questions related to the accuracy or integrity of any part of the work are appropriately investigated and resolved.

\section{Conflict of Interest}

None.

\section{Acknowledgement}

A part of this study has been presented as an oral paper in the state level pathology conference- MAPCON in September 2016 in Mumbai, India.

\section{References}

1 Haines M, Taylor CW, Fox H, Wells M. Haines and Taylor Obstetrical and Gynaecological Pathology. 5th edition. Edinburgh: Churchill Livingstone; 2003. Chapter 39, Pathology of pregnant uterus; pp. 1327-1357

2 Hustin J, Foidart JM, Lambotte R. Maternal vascular lesions in preeclampsia and intrauterine growth retardation: light microscopy and immunofluorescence. Placenta 1983;4(Spec No):489-498

3 Robertson WB, Khong TY, Brosens I, De Wolf F, Sheppard BL, Bonnar J. The placental bed biopsy: review from three European centers. Am J Obstet Gynecol 1986;155(02):401-412

4 Rushton DI, Dawson IM. The maternal autopsy. J Clin Pathol 1982; 35(09):909-921

5 Cunningham F, Leveno K, Bloom S, Hauth J, Rouse D. Williams Obstetrics. $23^{\text {rd }}$ edition. New York: McGraw- Hill Medical; 2010. Chapter 3, Implantation, Embryogenesis and Placental development; pp. 36-77

6 Flake GP, Moore AB, Sutton D, et al. The natural history of uterine leiomyomas: light and electron microscopic studies of fibroid phases, interstitial ischemia, inanosis, and reclamation. Obstet Gynecol Int 2013;2013:528376. Doi: 10.1155/2013/528376

7 Pijnenborg R, Vercruysse L, Hanssens M. The uterine spiral arteries in human pregnancy: facts and controversies. Placenta 2006; 27(9-10):939-958

8 Lyall F, Robson SC, Bulmer JN. Spiral artery remodeling and trophoblast invasion in preeclampsia and fetal growth restriction: relationship to clinical outcome. Hypertension 2013;62(06): 1046-1054

9 Jashnani KD, Rupani AB, Wani RJ. Maternal mortality: an autopsy audit. J Postgrad Med 2009;55(01):12-16

10 Jashnani KD, Chandekar SA, Pawar A, Dalal AR, Wani RJ. Hepatitis E infection and pregnancy: a fatal combination. Int J Basic Appl Med Sci. 2015;5(02):264-269

11 Redman CW, Sargent IL. Latest advances in understanding preeclampsia. Science 2005;308(5728):1592-1594

12 Burton GJ, Woods AW, Jauniaux E, Kingdom JC. Rheological and physiological consequences of conversion of the maternal spiral arteries for uteroplacental blood flow during human pregnancy. Placenta 2009;30(06):473-482

13 Burton GJ, Yung HW, Cindrova-Davies T, Charnock-Jones DS. Placental endoplasmic reticulum stress and oxidative stress in the pathophysiology of unexplained intrauterine growth restriction and early onset preeclampsia. Placenta 2009;30(Suppl A):S43-S48

14 Meekins JW, Pijnenborg R, Hanssens M, McFadyen IR, van Asshe A. A study of placental bed spiral arteries and trophoblast invasion in normal and severe pre-eclamptic pregnancies. $\mathrm{Br} \mathrm{J}$ Obstet Gynaecol 1994;101(08):669-674

15 Pijnenborg R, Anthony J, Davey DA, et al. Placental bed spiral arteries in the hypertensive disorders of pregnancy. Br J Obstet Gynaecol 1991;98(07):648-655

16 Avagliano L, Bulfamante GP, Morabito A, Marconi AM. Abnormal spiral artery remodelling in the decidual segment during pregnancy: from histology to clinical correlation. J Clin Pathol 2011;64 (12):1064-1068 